\title{
Global dynamics of some systems of higher-order rational difference equations
}

\author{
Abdul Qadeer Khan, Muhammad Naeem Qureshi and Qamar Din*
}

${ }^{*}$ Correspondence:

qamar.sms@gmail.com

Department of Mathematics,

University of Azad Jammu and

Kashmir, Muzaffarabad, Pakistan

\begin{abstract}
In the present work, we study the qualitative behavior of two systems of higher-order rational difference equations. More precisely, we study the local asymptotic stability, instability, global asymptotic stability of equilibrium points and rate of convergence of positive solutions of these systems. Our results considerably extend and improve some recent results in the literature. Some numerical examples are given to verify our theoretical results.
\end{abstract}

MSC: 39A10; 40A05

Keywords: system of difference equations; stability; global character; rate of convergence

\section{Introduction}

Recently, studying the qualitative behavior of difference equations and systems is a topic of great interest. Applications of discrete dynamical systems and difference equations have appeared recently in many areas such as ecology, population dynamics, queuing problems, statistical problems, stochastic time series, combinatorial analysis, number theory, geometry, electrical networks, neural networks, quanta in radiation, genetics in biology, economics, psychology, sociology, physics, engineering, economics, probability theory and resource management. Unfortunately, these are only considered as the discrete analogs of differential equations. It is a well-known fact that difference equations appeared much earlier than differential equations and were instrumental in paving the way for the development of the latter. It is only recently that difference equations have started receiving the attention they deserve. Perhaps this is largely due to the advent of computers where differential equations are solved by using their approximate difference equation formulations. The theory of discrete dynamical systems and difference equations developed greatly during the last twenty-five years of the twentieth century. The theory of difference equations occupies a central position in applicable analysis. There is no doubt that the theory of difference equations will continue to play an important role in mathematics as a whole. Nonlinear difference equations of order greater than one are of paramount importance in applications. It is very interesting to investigate the behavior of solutions of a system of higher-order rational difference equations and to discuss the local asymptotic stability of their equilibrium points. Systems of rational difference equations have been studied by several authors. Especially there has been a great interest in the study of the attractivity of the solutions of such systems. For more results on the qualitative behavior of nonlinear difference equations, we refer the interested reader to [1-19].

C2013 Khan et al: licensee Springer. This is an Open Access article distributed under the terms of the Creative Commons Attribution License (http://creativecommons.org/licenses/by/2.0), which permits unrestricted use, distribution, and reproduction in any medium, provided the original work is properly cited. 
Zhang et al. [20] studied the dynamics of a system of rational third-order difference equations

$$
x_{n+1}=\frac{x_{n-2}}{B+y_{n} y_{n-1} y_{n-2}}, \quad y_{n+1}=\frac{y_{n-2}}{A+x_{n} x_{n-1} x_{n-2}} .
$$

Din et al. [11] investigated the dynamics of a system of fourth-order rational difference equations

$$
x_{n+1}=\frac{\alpha x_{n-3}}{\beta+\gamma y_{n} y_{n-1} y_{n-2} y_{n-3}}, \quad y_{n+1}=\frac{\alpha_{1} y_{n-3}}{\beta_{1}+\gamma_{1} x_{n} x_{n-1} x_{n-2} x_{n-3}} .
$$

To be motivated by the above studies, our aim in this paper is to investigate the qualitative behavior of the following $(k+1) t h$-order systems of rational difference equations:

$$
x_{n+1}=\frac{\alpha x_{n-k}}{\beta+\gamma \prod_{i=0}^{k} y_{n-i}}, \quad y_{n+1}=\frac{\alpha_{1} y_{n-k}}{\beta_{1}+\gamma_{1} \prod_{i=0}^{k} x_{n-i}}, \quad n=0,1, \ldots
$$

where the parameters $\alpha, \beta, \gamma, \alpha_{1}, \beta_{1}, \gamma_{1}$ and initial conditions $x_{0}, x_{-1}, \ldots, x_{-k}, y_{0}, y_{-1}, \ldots, y_{-k}$ are positive real numbers, and

$$
x_{n+1}=\frac{a y_{n-k}}{b+c \prod_{i=0}^{k} x_{n-i}}, \quad y_{n+1}=\frac{a_{1} x_{n-k}}{b_{1}+c_{1} \prod_{i=0}^{k} y_{n-i}}, \quad n=0,1, \ldots,
$$

where the parameters $a, b, c, a_{1}, b_{1}, c_{1}$ and initial conditions $x_{0}, x_{-1}, \ldots, x_{-k}, y_{0}, y_{-1}, \ldots, y_{-k}$ are positive real numbers. This paper is a natural extension of [11, 20, 21].

Let us consider $(2 k+2)$-dimensional discrete dynamical system of the form

$$
\begin{aligned}
& x_{n+1}=f\left(x_{n}, x_{n-1}, \ldots, x_{n-k}, y_{n}, y_{n-1}, \ldots, y_{n-k}\right), \\
& y_{n+1}=g\left(x_{n}, x_{n-1}, \ldots, x_{n-k}, y_{n}, y_{n-1}, \ldots, y_{n-k}\right), \quad n=0,1, \ldots,
\end{aligned}
$$

where $f: I^{k+1} \times J^{k+1} \rightarrow I$ and $g: I^{k+1} \times J^{k+1} \rightarrow J$ are continuously differentiable functions and $I, J$ are some intervals of real numbers. Furthermore, a solution $\left\{\left(x_{n}, y_{n}\right)\right\}_{n=-k}^{\infty}$ of system (3) is uniquely determined by initial conditions $\left(x_{i}, y_{i}\right) \in I \times J$ for $i \in\{-k,-k+$ $1, \ldots,-1,0\}$. Along with system (3), we consider the corresponding vector map $F=$ $\left(f, x_{n}, x_{n-1}, \ldots, x_{n-k}, g, y_{n}, y_{n-1}, \ldots, y_{n-k}\right)$. An equilibrium point of $(3)$ is a point $(\bar{x}, \bar{y})$ that satisfies

$$
\begin{aligned}
& \bar{x}=f(\bar{x}, \bar{x}, \ldots, \bar{x}, \bar{y}, \bar{y}, \ldots, \bar{y}), \\
& \bar{y}=g(\bar{x}, \bar{x}, \ldots, \bar{x}, \bar{y}, \bar{y}, \ldots, \bar{y}) .
\end{aligned}
$$

The point $(\bar{x}, \bar{y})$ is also called a fixed point of the vector map $F$.

Definition 1 Let $(\bar{x}, \bar{y})$ be an equilibrium point of system (3).

(i) An equilibrium point $(\bar{x}, \bar{y})$ is said to be stable if for every $\varepsilon>0$ there exists $\delta>0$ such that for every initial condition $\left(x_{i}, y_{i}\right), i \in\{-k,-k+1, \ldots,-1,0\}$, $\left\|\sum_{i=-k}^{0}\left(x_{i}, y_{i}\right)-(\bar{x}, \bar{y})\right\|<\delta$ implies $\left\|\left(x_{n}, y_{n}\right)-(\bar{x}, \bar{y})\right\|<\varepsilon$ for all $n>0$, where $\|\cdot\|$ is the usual Euclidian norm in $\mathbb{R}^{2}$. 
(ii) An equilibrium point $(\bar{x}, \bar{y})$ is said to be unstable if it is not stable.

(iii) An equilibrium point $(\bar{x}, \bar{y})$ is said to be asymptotically stable if there exists $\eta>0$ such that $\left\|\sum_{i=-k}^{0}\left(x_{i}, y_{i}\right)-(\bar{x}, \bar{y})\right\|<\eta$ and $\left(x_{n}, y_{n}\right) \rightarrow(\bar{x}, \bar{y})$ as $n \rightarrow \infty$.

(iv) An equilibrium point $(\bar{x}, \bar{y})$ is called a global attractor if $\left(x_{n}, y_{n}\right) \rightarrow(\bar{x}, \bar{y})$ as $n \rightarrow \infty$.

(v) An equilibrium point $(\bar{x}, \bar{y})$ is called an asymptotic global attractor if it is a global attractor and stable.

Definition 2 Let $(\bar{x}, \bar{y})$ be an equilibrium point of the map

$$
F=\left(f, x_{n}, x_{n-1}, \ldots, x_{n-k}, g, y_{n}, y_{n-1}, \ldots, y_{n-k}\right),
$$

where $f$ and $g$ are continuously differentiable functions at $(\bar{x}, \bar{y})$. The linearized system of (3) about the equilibrium point $(\bar{x}, \bar{y})$ is

$$
X_{n+1}=F\left(X_{n}\right)=F_{J} X_{n},
$$

where

$$
X_{n}=\left(\begin{array}{c}
x_{n} \\
x_{n-1} \\
\vdots \\
x_{n-k} \\
y_{n} \\
y_{n-1} \\
\vdots \\
y_{n-k}
\end{array}\right)
$$

and $F_{J}$ is the Jacobian matrix of system (3) about the equilibrium point $(\bar{x}, \bar{y})$.

Lemma 1 [22] Assume that $X_{n+1}=F\left(X_{n}\right), n=0,1, \ldots$, is a system of difference equations and $\bar{X}$ is the fixed point of $F$. If all eigenvalues of the Jacobian matrix $J_{F}$ about $\bar{X}$ lie inside an open unit disk $|\lambda|<1$, then $\bar{X}$ is locally asymptotically stable. If one of them has norm greater than one, then $\bar{X}$ is unstable.

Lemma 2 [23] Assume that $X_{n+1}=F\left(X_{n}\right), n=0,1, \ldots$, is a system of difference equations and $\bar{X}$ is the equilibrium point of this system. The characteristic polynomial of this system about the equilibrium point $\bar{X}$ is $P(\lambda)=a_{0} \lambda^{n}+a_{1} \lambda^{n-1}+\cdots+a_{n-1} \lambda+a_{n}=0$, with real coefficients and $a_{0}>0$. Then all roots of the polynomial $P(\lambda)$ lie inside the open unit disk $|\lambda|$ if and only if $\Delta_{k}>0$ for $k=0,1, \ldots$, where $\Delta_{k}$ is the principal minor of order $k$ of the $n \times n$ matrix

$$
\Delta_{n}=\left(\begin{array}{ccccc}
a_{1} & a_{3} & a_{5} & \ldots & 0 \\
a_{0} & a_{2} & a_{4} & \ldots & 0 \\
0 & a_{1} & a_{3} & \ldots & 0 \\
\vdots & \vdots & \ddots & \vdots & \\
0 & 0 & 0 & \ldots & a_{n}
\end{array}\right) .
$$


Let us consider a system of difference equations

$$
X_{n+1}=(A+B(n)) X_{n}
$$

where $X_{n}$ is an $m$-dimensional vector, $A \in C^{m \times m}$ is a constant matrix, and $B: \mathbb{Z}^{+} \rightarrow C^{m \times m}$ is a matrix function satisfying

$$
\|B(n)\| \rightarrow 0
$$

as $n \rightarrow \infty$, where $\|\cdot\|$ denotes any matrix norm which is associated with the vector norm

$$
\|(x, y)\|=\sqrt{x^{2}+y^{2}}
$$

Proposition 1 (Perron's theorem)[24] Suppose that condition(6) holds. If $X_{n}$ is a solution of (5), then either $X_{n}=0$ for all large $n$ or

$$
\rho=\lim _{n \rightarrow \infty}\left(\left\|X_{n}\right\|\right)^{1 / n}
$$

exists and is equal to the modulus of one of the eigenvalues of matrix $A$.

Proposition 2 [24] Suppose that condition (6) holds. If $X_{n}$ is a solution of (5), then either $X_{n}=0$ for all large $n$ or

$$
\rho=\lim _{n \rightarrow \infty} \frac{\left\|X_{n+1}\right\|}{\left\|X_{n}\right\|}
$$

exists and is equal to the modulus of one of the eigenvalues of matrix $A$.

\section{On the system $x_{n+1}=\frac{\alpha x_{n-k}}{\beta+\gamma \prod_{i=0}^{k} y_{n-i}}, y_{n+1}=\frac{\alpha_{1} y_{n-k}}{\beta_{1}+\gamma_{1} \prod_{i=0}^{k} x_{n-i}}$}

In this section, we shall investigate the qualitative behavior of system $(1)$. Let $(\bar{x}, \bar{y})$ be an equilibrium point of system (1), then for $\alpha>\beta$ and $\alpha_{1}>\beta_{1}$, system (1) has two positive equilibrium points $P_{0}=(0,0), P_{1}=(A, B)$, where $A=\left(\frac{\alpha_{1}-\beta_{1}}{\gamma_{1}}\right)^{\frac{1}{k+1}}$ and $B=\left(\frac{\alpha-\beta}{\gamma}\right)^{\frac{1}{k+1}}$.

To construct the corresponding linearized form of system (1), we consider the following transformation:

$$
\left(x_{n}, x_{n-1}, x_{n-2}, \ldots, x_{n-k}, y_{n}, y_{n-1}, \ldots, y_{n-k}\right) \mapsto\left(f, f_{1}, \ldots, f_{n-k}, g, g_{1}, \ldots, g_{n-k}\right),
$$

where $f=\frac{\alpha x_{n-k}}{\beta+\gamma \prod_{i=0}^{k} y_{n-i}}, f_{1}=x_{n}, f_{2}=x_{n-1}, \ldots, f_{n-k}=x_{n-(k-1)}$ and $g=\frac{\alpha_{1} y_{n-k}}{\beta_{1}+\gamma_{1} \prod_{i=0}^{k} x_{n-i}}, g_{1}=y_{n}$, $g_{2}=y_{n-1}, \ldots, g_{n-k}=y_{n-(k-1)}$. The Jacobian matrix about the fixed point $(\bar{x}, \bar{y})$ under the 
transformation (9) is given by

$$
F_{J}(\bar{x}, \bar{y})=\left(\begin{array}{cccccccccc}
0 & 0 & \ldots & 0 & A & B & B & \ldots & B & B \\
1 & 0 & \ldots & 0 & 0 & 0 & 0 & \ldots & 0 & 0 \\
\vdots & \vdots & \ddots & \vdots & \vdots & \vdots & \ddots & \vdots & \vdots & \vdots \\
0 & 0 & \ldots & 1 & 0 & 0 & 0 & \ldots & 0 & 0 \\
C & C & \ldots & C & C & 0 & 0 & \ldots & 0 & D \\
0 & 0 & \ldots & 0 & 0 & 1 & 0 & \ldots & 0 & 0 \\
\vdots & \vdots & \ddots & \vdots & \vdots & \vdots & \ddots & \vdots & \vdots & \vdots \\
0 & 0 & \ldots & 0 & 0 & 0 & 0 & \ldots & 1 & 0
\end{array}\right),
$$

where $A=\frac{\alpha}{\beta+\gamma \bar{y}^{k+1}}, B=-\frac{\alpha \gamma \bar{x} \bar{y}^{k}}{\left(\beta+\gamma \bar{y}^{k+1}\right)^{2}}, C=-\frac{\alpha_{1} \gamma_{1} \overline{\bar{y}} \bar{x}^{k}}{\left(\beta_{1}+\gamma_{1} \bar{x}^{k+1}\right)^{2}}$ and $D=\frac{\alpha_{1}}{\beta_{1}+\gamma_{1} \bar{x}^{k+1}}$.

Theorem 1 Let $\alpha<\beta$ and $\alpha_{1}<\beta_{1}$, then every solution $\left\{\left(x_{n}, y_{n}\right)\right\}$ of system (1) is bounded.

Proof It is easy to verify that

$$
\begin{aligned}
& 0 \leq x_{n} \leq\left(\frac{\alpha}{\beta}\right)^{m+1} x_{-k} \quad \text { if } n=(k+1) m+1, \\
& 0 \leq x_{n} \leq\left(\frac{\alpha}{\beta}\right)^{m+1} x_{1-k} \quad \text { if } n=(k+1) m+2, \\
& \vdots \\
& 0 \leq x_{n} \leq\left(\frac{\alpha}{\beta}\right)^{m+1} x_{-1} \quad \text { if } n=(k+1) m+k \\
& 0 \leq x_{n} \leq\left(\frac{\alpha}{\beta}\right)^{m+1} x_{0} \quad \text { if } n=(k+1) m+(k+1),
\end{aligned}
$$

and

$$
\begin{aligned}
& 0 \leq y_{n} \leq\left(\frac{\alpha_{1}}{\beta_{1}}\right)^{m+1} y_{-k} \quad \text { if } n=(k+1) m+1, \\
& 0 \leq y_{n} \leq\left(\frac{\alpha_{1}}{\beta_{1}}\right)^{m+1} y_{1-k} \quad \text { if } n=(k+1) m+2, \\
& \vdots \\
& 0 \leq y_{n} \leq\left(\frac{\alpha_{1}}{\beta_{1}}\right)^{m+1} y_{-1} \quad \text { if } n=(k+1) m+k, \\
& 0 \leq y_{n} \leq\left(\frac{\alpha_{1}}{\beta_{1}}\right)^{m+1} y_{0} \quad \text { if } n=(k+1) m+k+1 .
\end{aligned}
$$

Take $\delta_{1}=\max \left\{x_{-k}, \ldots, x_{0}\right\}$ and $\delta_{2}=\max \left\{y_{-k}, \ldots, y_{0}\right\}$. Then $0 \leq x_{n}<\delta_{1}$ and $0 \leq y_{n}<\delta_{2}$ for all $n=0,1,2, \ldots$.

Theorem 2 The equilibrium point $P_{0}$ of system (1) is locally asymptotically stable. 
Proof The linearized system of (1) about the equilibrium point $(0,0)$ is given by

$$
X_{n+1}=F_{J}(0,0) X_{n}
$$

where

$$
X_{n}=\left(\begin{array}{c}
x_{n} \\
x_{n-1} \\
\vdots \\
x_{n-k} \\
y_{n} \\
y_{n-1} \\
\vdots \\
y_{n-k}
\end{array}\right)
$$

and

$$
E=F_{J}\left(P_{0}\right)=\left(e_{i j}\right)_{(2 k+2) \times(2 k+2)}=\left(\begin{array}{cccccccccc}
0 & 0 & \ldots & 0 & \frac{\alpha}{\beta} & 0 & 0 & \ldots & 0 & 0 \\
1 & 0 & \ldots & 0 & 0 & 0 & 0 & \ldots & 0 & 0 \\
\vdots & \vdots & \ddots & \vdots & \vdots & \vdots & \ddots & \vdots & \vdots & \vdots \\
0 & 0 & \ldots & 1 & 0 & 0 & 0 & \ldots & 0 & 0 \\
0 & 0 & \ldots & 0 & 0 & 0 & 0 & \ldots & 0 & \frac{\alpha_{1}}{\beta_{1}} \\
0 & 0 & \ldots & 0 & 0 & 1 & 0 & \ldots & 0 & 0 \\
\vdots & \vdots & \ddots & \vdots & \vdots & \vdots & \ddots & \vdots & \vdots & \vdots \\
0 & 0 & \ldots & 0 & 0 & 0 & 0 & \ldots & 1 & 0
\end{array}\right) .
$$

Let $\lambda_{1}, \lambda_{2}, \ldots, \lambda_{2 k+2}$ denote the $2 k+2$ eigenvalues of matrix $E$. Let $D=\operatorname{diag}\left(d_{1}, d_{2}, \ldots, d_{2 k+2}\right)$ be a diagonal matrix, where $d_{1}=d_{k+2}=1, d_{1+m}=d_{k+2+m}=1-m \epsilon, 1 \leq m \leq k$, and

$$
\epsilon=\min \left\{\frac{1}{k}, \frac{1}{k}\left(1-\frac{\alpha}{\beta}\right), \frac{1}{k}\left(1-\frac{\alpha_{1}}{\beta_{1}}\right)\right\} .
$$

Clearly, $D$ is invertible. Computing $D E D^{-1}$, we obtain

$$
D E D^{-1}=\left(\begin{array}{cccccccccc}
0 & 0 & \ldots & 0 & \frac{\alpha}{\beta} d_{1} d_{k+1}^{-1} & 0 & 0 & \ldots & 0 & 0 \\
d_{2} d_{1}^{-1} & 0 & \ldots & 0 & 0 & 0 & 0 & \ldots & 0 & 0 \\
\vdots & \vdots & \ldots & \vdots & \vdots & \vdots & \ddots & \vdots & \vdots & \vdots \\
0 & 0 & \ldots & d_{k+1} d_{k}^{-1} & 0 & 0 & 0 & \ldots & 0 & 0 \\
0 & 0 & \ldots & 0 & 0 & 0 & 0 & \ldots & 0 & \frac{\alpha_{1}}{\beta_{1}} d_{k+2} d_{2 k+2}^{-1} \\
0 & 0 & \ldots & 0 & 0 & d_{k+3} d_{k+2}^{-1} & 0 & \ldots & 0 & 0 \\
\vdots & \vdots & \ddots & \vdots & \vdots & \vdots & \ddots & \vdots & \vdots & \vdots \\
0 & 0 & \ldots & 0 & 0 & 0 & 0 & \ldots & d_{2 k+2} d_{2 k+1}^{-1} & 0
\end{array}\right)
$$

We obtain the following two inequalities:

$$
0<d_{k+1}<d_{k}<\cdots<d_{2}, \quad 0<d_{2 k+2}<d_{2 k+1}<\cdots<d_{k+3},
$$


which implies that

$$
d_{2} d_{1}^{-1}<1, \quad d_{3} d_{2}^{-1}<1, \quad \ldots, \quad d_{k+1} d_{k}^{-1}<1,
$$

and

$$
d_{k+3} d_{k+2}^{-1}<1, \quad d_{k+4} d_{k+3}^{-1}<1, \quad \ldots, \quad d_{2 k+2} d_{2 k+1}^{-1}<1 .
$$

Furthermore,

$$
\frac{\alpha}{\beta} d_{1} d_{k+1}^{-1}=\frac{\alpha}{\beta} d_{k+1}^{-1}=\frac{\alpha}{\beta} \frac{1}{1-k \epsilon}<1,
$$

and

$$
\frac{\alpha_{1}}{\beta_{1}} d_{k+2} d_{2 k+2}^{-1}=\frac{\alpha_{1}}{\beta_{1}} d_{2 k+2}^{-1}=\frac{\alpha_{1}}{\beta_{1}} \frac{1}{1-k \epsilon}<1 .
$$

It is a well-known fact that $E$ has the same eigenvalues as $D E D^{-1}$. Hence, we obtain

$$
\begin{aligned}
& \max _{1 \leq m \leq 2 k+2}\left|\lambda_{m}\right| \\
& =\left\|D E D^{-1}\right\| \\
& =\max \left\{d_{2} d_{1}^{-1}, \ldots, d_{k+1} d_{k}^{-1}, d_{k+3} d_{k+2}^{-1}, \ldots, d_{2 k+2} d_{2 k+1}^{-1}, \frac{\alpha}{\beta} d_{1} d_{k+1}^{-1}, \frac{\alpha_{1}}{\beta_{1}} d_{k+2} d_{2 k+2}^{-1}\right\}<1 .
\end{aligned}
$$

Hence, the equilibrium point $P_{0}$ of system (1) is locally asymptotically stable.

Theorem 3 The positive equilibrium point $P_{1}$ of system (1) is unstable.

Proof The linearized system of (1) about the equilibrium point $P_{1}$ is given by

$$
X_{n+1}=F_{J}\left(P_{1}\right) X_{n},
$$

where

$$
X_{n}=\left(\begin{array}{c}
x_{n} \\
x_{n-1} \\
\vdots \\
x_{n-k} \\
y_{n} \\
y_{n-1} \\
\vdots \\
y_{n-k}
\end{array}\right)
$$


and

$$
F_{J}\left(P_{1}\right)=\left(\begin{array}{cccccccccc}
0 & 0 & \ldots & 0 & 1 & L & L & \ldots & L & L \\
1 & 0 & \ldots & 0 & 0 & 0 & 0 & \ldots & 0 & 0 \\
\vdots & \vdots & \ddots & \vdots & \vdots & \vdots & \ddots & \vdots & \vdots & \vdots \\
0 & 0 & \ldots & 1 & 0 & 0 & 0 & \ldots & 0 & 0 \\
M & M & \ldots & M & M & 0 & 0 & \ldots & 0 & 1 \\
0 & 0 & \ldots & 0 & 0 & 1 & 0 & \ldots & 0 & 0 \\
\vdots & \vdots & \ddots & \vdots & \vdots & \vdots & \ddots & \vdots & \vdots & \vdots \\
0 & 0 & \ldots & 0 & 0 & 0 & 0 & \ldots & 1 & 0
\end{array}\right),
$$

where $L=-\left(\frac{\gamma}{\gamma_{1}}\right) \frac{1}{k+1} \frac{\left(\alpha_{1}-\beta_{1}\right)^{\frac{1}{k+1}}(\alpha-\beta) \frac{k}{k+1}}{\alpha}$ and $M=-\left(\frac{\gamma_{1}}{\gamma}\right)^{\frac{1}{k+1}} \frac{(\alpha-\beta)^{\frac{1}{k+1}}\left(\alpha_{1}-\beta_{1}\right) \frac{k}{k+1}}{\alpha_{1}}$. The characteristic polynomial of $F_{J}\left(P_{1}\right)$ is given by

$$
\begin{aligned}
P(\lambda)= & \lambda^{2 k+2}-L M\left[\lambda^{2 k}+2 \lambda^{2 k-1}+\cdots+k \lambda^{k+1}+(k+1) \lambda^{k}\right. \\
& \left.+k \lambda^{k-1}+(k-1) \lambda^{k-2}+\cdots+1\right]+2 \lambda^{k+1}+1 .
\end{aligned}
$$

From (10), we have

$$
\Delta_{(2 k+2) \times(2 k+2)}=\left(\begin{array}{ccccc}
-L M & -3 L M & -5 L M & \ldots & 0 \\
1 & -2 L M & -4 L M & \ldots & 0 \\
0 & -L M & -3 L M & \ldots & 0 \\
\vdots & \vdots & \ddots & \vdots & \\
0 & 0 & 0 & \ldots & 1-L M
\end{array}\right) .
$$

It is clear that not all of $\Delta_{(2 k+2) \times(2 k+2)}>0$. Therefore, by Lemma 1, the unique positive equilibrium point $(\bar{x}, \bar{y})=\left(\left(\frac{\alpha_{1}-\beta_{1}}{\gamma_{1}}\right)^{\frac{1}{k+1}},\left(\frac{\alpha-\beta}{\gamma}\right)^{\frac{1}{k+1}}\right)$ is unstable.

Theorem 4 Let $\alpha>\beta$ and $\alpha_{1}>\beta_{1}$, and let $\left\{\left(x_{n}, y_{n}\right)\right\}$ be a solution of system (1). Then, for $i=0,1, \ldots, k$, the following statements are true:

(i) If $\left(x_{i}, y_{i}\right) \in\left(0,\left(\frac{\alpha_{1}-\beta_{1}}{\gamma_{1}}\right)^{\frac{1}{k+1}}\right) \times\left(\left(\frac{\alpha-\beta}{\gamma}\right)^{\frac{1}{k+1}}, \infty\right)$, then

$$
\left(x_{n}, y_{n}\right) \in\left(0,\left(\frac{\alpha_{1}-\beta_{1}}{\gamma_{1}}\right)^{\frac{1}{k+1}}\right) \times\left(\left(\frac{\alpha-\beta}{\gamma}\right)^{\frac{1}{k+1}}, \infty\right) \text {. }
$$

(ii) If $\left(x_{i}, y_{i}\right) \in\left(\left(\frac{\alpha_{1}-\beta_{1}}{\gamma_{1}}\right)^{\frac{1}{k+1}}, \infty\right) \times\left(0,\left(\frac{\alpha-\beta}{\gamma}\right)^{\frac{1}{k+1}}\right)$, then

$$
\left(x_{n}, y_{n}\right) \in\left(\left(\frac{\alpha_{1}-\beta_{1}}{\gamma_{1}}\right)^{\frac{1}{k+1}}, \infty\right) \times\left(0,\left(\frac{\alpha-\beta}{\gamma}\right)^{\frac{1}{k+1}}\right) \text {. }
$$

Proof It follows from induction.

Theorem 5 Let $\alpha<\beta$ and $\alpha_{1}<\beta_{1}$, then the equilibrium point $P_{0}$ of system (1) is globally asymptotically stable.

Proof For $\alpha<\beta$ and $\alpha_{1}<\beta_{1}$, from Theorem 2, $P_{0}$ is locally asymptotically stable. From Theorem 1, every positive solution $\left(x_{n}, y_{n}\right)$ is bounded, i.e., $0 \leq x_{n} \leq \mu$ and $0 \leq y_{n} \leq v$ for 
all $n=0,1,2, \ldots$, where $\mu=\max \left\{x_{-k}, x_{-k+1}, \ldots, x_{-1}, x_{0}\right\}$ and $v=\max \left\{y_{-k}, y_{-k+1}, \ldots, y_{-1}, y_{0}\right\}$. So, it is sufficient to prove that $\left\{\left(x_{n}, y_{n}\right)\right\}$ is decreasing. From system (1), one has

$$
x_{n+1}=\frac{\alpha x_{n-k}}{\beta+\gamma \prod_{i=0}^{k} y_{n-i}} \leq \frac{\alpha x_{n-k}}{\beta}<x_{n-k} .
$$

This implies that $x_{(k+1) n+1}<x_{(k+1) n-k}$ and $x_{(k+1) n+(k+2)}<x_{(k+1) n+1}$. Hence, the subsequences

$$
\left\{x_{(k+1) n+1}\right\}, \quad\left\{x_{(k+1) n+2}\right\}, \quad \ldots, \quad\left\{x_{(k+1) n+k}\right\}, \quad\left\{x_{(k+1) n+(k+1)}\right\}
$$

are decreasing, i.e., the sequence $\left\{x_{n}\right\}$ is decreasing. Also,

$$
y_{n+1}=\frac{\alpha y_{n-k}}{\beta+\gamma \prod_{i=0}^{k} x_{n-i}} \leq \frac{\alpha y_{n-k}}{\beta}<y_{n-k} .
$$

This implies that $y_{(k+1) n+1}<y_{(k+1) n-k}$ and $y_{(k+1) n+(k+2)}<y_{(k+1) n+1}$. Hence, the subsequences

$$
\left\{y_{(k+1) n+1}\right\}, \quad\left\{y_{(k+1) n+2}\right\}, \quad \ldots, \quad\left\{y_{(k+1) n+k}\right\}, \quad\left\{y_{(k+1) n+(k+1)}\right\}
$$

are decreasing, i.e., the sequence $\left\{y_{n}\right\}$ is decreasing. Hence, $\lim _{n \rightarrow \infty} x_{n}=\lim _{n \rightarrow \infty} y_{n}=0$

Theorem 6 Let $\alpha>\beta$ and $\alpha_{1}>\beta_{1}$. Then, for a solution $\left\{\left(x_{n}, y_{n}\right)\right\}$ of system (1), the following statements are true:

(i) If $x_{n} \rightarrow 0$, then $y_{n} \rightarrow \infty$.

(ii) If $y_{n} \rightarrow 0$, then $x_{n} \rightarrow \infty$.

\subsection{Rate of convergence}

We investigate the rate of convergence of a solution that converges to the equilibrium point $P_{0}$ of system (1).

Assume that $\lim _{n \rightarrow \infty} x_{n}=\bar{x}$ and $\lim _{n \rightarrow \infty} x_{n}=\bar{y}$. First we will find a system of limiting equations for the map $F$. The error terms are given as

$$
\begin{aligned}
& x_{n+1}-\bar{x}=\sum_{i=0}^{k} A_{i}\left(x_{n-i}-\bar{x}\right)+\sum_{i=0}^{k} B_{i}\left(y_{n-i}-\bar{y}\right), \\
& y_{n+1}-\bar{y}=\sum_{i=0}^{k} C_{i}\left(x_{n-i}-\bar{x}\right)+\sum_{i=0}^{k} D_{i}\left(y_{n-i}-\bar{y}\right) .
\end{aligned}
$$

Set $e_{n}^{1}=x_{n}-\bar{x}$ and $e_{n}^{2}=y_{n}-\bar{y}$, one has

$$
e_{n+1}^{1}=\sum_{i=0}^{k} A_{i} e_{n-i}^{1}+\sum_{i=0}^{k} B_{i} e_{n-i}^{2}, \quad e_{n+1}^{2}=\sum_{i=0}^{k} C_{i} e_{n-i}^{1}+\sum_{i=0}^{k} D_{i} e_{n-i}^{2},
$$

where $A_{i}=0$ for $i \in\{0,1, \ldots, k-1\}$,

$$
\begin{aligned}
& A_{k}=\frac{\alpha}{\beta+\gamma \prod_{i=0}^{k} y_{n-i}}, \quad B_{0}=-\frac{\alpha \gamma \bar{x} y_{n-1} y_{n-2} \cdots y_{n-k}}{\left(\beta+\gamma \prod_{i=0}^{k} y_{n-i}\right)\left(\beta+\gamma \bar{y}^{k+1}\right)}, \\
& B_{1}=-\frac{\alpha \gamma \bar{x} \bar{y} y_{n-2} y_{n-3} \cdots y_{n-k}}{\left(\beta+\gamma \prod_{i=0}^{k} y_{n-i}\right)\left(\beta+\gamma \bar{y}^{k+1}\right)}, \quad B_{2}=-\frac{\alpha \gamma \bar{x} \bar{y}^{2} y_{n-3} \cdots y_{n-k}}{\left(\beta+\gamma \prod_{i=0}^{k} y_{n-i}\right)\left(\beta+\gamma \bar{y}^{k+1}\right)},
\end{aligned}
$$




$$
\begin{aligned}
& B_{k-1}=-\frac{\alpha \gamma \bar{x} \bar{y}^{k-1} y_{n-k}}{\left(\beta+\gamma \prod_{i=0}^{k} y_{n-i}\right)\left(\beta+\gamma \bar{y}^{k+1}\right)}, \quad B_{k}=-\frac{\alpha \gamma \bar{x} \bar{y}^{k}}{\left(\beta+\gamma \prod_{i=0}^{k} y_{n-i}\right)\left(\beta+\gamma \bar{y}^{k+1}\right)}, \\
& C_{0}=-\frac{\alpha_{1} \gamma_{1} \bar{y} x_{n-1} x_{n-2} \cdots x_{n-k}}{\left(\beta_{1}+\gamma_{1} \prod_{i=0}^{k} x_{n-i}\right)\left(\beta_{1}+\gamma_{1} \bar{x}^{k+1}\right)}, \\
& C_{1}=-\frac{\alpha_{1} \gamma_{1} \bar{y} \bar{x} x_{n-2} x_{n-3} \cdots x_{n-k}}{\left(\beta_{1}+\gamma_{1} \prod_{i=0}^{k} x_{n-i}\right)\left(\beta_{1}+\gamma_{1} \bar{x}^{k+1}\right)}, \\
& C_{2}=-\frac{\alpha_{1} \gamma_{1} \bar{y} \bar{x}^{2} x_{n-3} \cdots x_{n-k}}{\left(\beta_{1}+\gamma_{1} \prod_{i=0}^{k} x_{n-i}\right)\left(\beta_{1}+\gamma_{1} \bar{x}^{k+1}\right)}, \quad \ldots, \\
& C_{k-1}=-\frac{\alpha_{1} \gamma_{1} \bar{y} \bar{x}^{k-1} x_{n-k}}{\left(\beta_{1}+\gamma_{1} \prod_{i=0}^{k} x_{n-i}\right)\left(\beta_{1}+\gamma_{1} \bar{x}^{k+1}\right)}, \quad C_{k}=-\frac{\alpha_{1} \bar{x}^{k}}{\left(\beta_{1}+\gamma_{1} \prod_{i=0}^{k} x_{n-i}\right)\left(\beta_{1}+\gamma_{1} \bar{x}^{k+1}\right)}, \\
& D_{i}=0 \quad \text { for } i \in\{0,1, \ldots, k-1\} \quad \text { and } \quad D_{k}=\frac{\alpha_{1}}{\beta_{1}+\gamma_{1} \prod_{i=0}^{k} x_{n-i}} .
\end{aligned}
$$

Taking the limits, we obtain $\lim _{n \rightarrow \infty} A_{i}=0$ for $i \in\{0,1, \ldots, k-1\}, \lim _{n \rightarrow \infty} A_{k}=\frac{\alpha}{\beta+\gamma \bar{y}^{k+1}}$, $\lim _{n \rightarrow \infty} B_{i}=-\frac{\alpha \gamma \bar{x} \bar{y}^{k}}{\left(\beta+\gamma \bar{y}^{k+1}\right)^{2}}$ for $i \in\{0,1, \ldots, k\}, \lim _{n \rightarrow \infty} C_{i}=-\frac{\alpha_{1} \gamma_{1} \bar{y} \bar{x}^{k}}{\left(\beta_{1}+\gamma_{1} \bar{x}^{k+1}\right)^{2}}$ for $i \in\{0,1, \ldots, k\}$, $\lim _{n \rightarrow \infty} D_{i}=0$ for $i \in\{0,1, \ldots, k-1\}$ and $\lim _{n \rightarrow \infty} D_{k}=\frac{\alpha_{1}}{\beta_{1}+\gamma_{1} \bar{x}^{k+1}}$. Hence, the limiting system of error terms at $(\bar{x}, \bar{y})=(0,0)$ can be written as

$$
E_{n+1}=K E_{n}
$$

where

$$
\begin{aligned}
& E_{n}=\left(\begin{array}{c}
e_{n}^{1} \\
e_{n-1}^{1} \\
\vdots \\
e_{n-k}^{1} \\
e_{n}^{2} \\
e_{n-1}^{2} \\
\vdots \\
e_{n-k}^{2}
\end{array}\right) \\
& K=\left(\begin{array}{cccccccccc}
0 & 0 & \ldots & 0 & \frac{\alpha}{\beta} & 0 & 0 & \ldots & 0 & 0 \\
1 & 0 & \ldots & 0 & 0 & 0 & 0 & \ldots & 0 & 0 \\
\vdots & \vdots & \ddots & \vdots & \vdots & \vdots & \ddots & \vdots & \vdots & \vdots \\
0 & 0 & \ldots & 1 & 0 & 0 & 0 & \ldots & 0 & 0 \\
0 & 0 & \ldots & 0 & 0 & 0 & 0 & \ldots & 0 & \frac{\alpha_{1}}{\beta_{1}} \\
0 & 0 & \ldots & 0 & 0 & 1 & 0 & \ldots & 0 & 0 \\
\vdots & \vdots & \ddots & \vdots & \vdots & \vdots & \ddots & \vdots & \vdots & \vdots \\
0 & 0 & \ldots & 0 & 0 & 0 & 0 & \ldots & 1 & 0
\end{array}\right),
\end{aligned}
$$

which is similar to the linearized system of $(1)$ about the equilibrium point $(\bar{x}, \bar{y})=(0,0)$. Using proposition (1), one has the following result.

Theorem 7 Assume that $\left\{\left(x_{n}, y_{n}\right)\right\}$ is a positive solution of system (1) such that $\lim _{n \rightarrow \infty} x_{n}=$ $\bar{x}$, and $\lim _{n \rightarrow \infty} y_{n}=\bar{y}$, where $(\bar{x}, \bar{y})=(0,0)$. Then the error vector $E_{n}$ of every solution of $(1)$ 
satisfies both of the following asymptotic relations:

$$
\lim _{n \rightarrow \infty}\left(\left\|E_{n}\right\|\right)^{\frac{1}{n}}=\left|\lambda F_{J}(\bar{x}, \bar{y})\right|, \quad \lim _{n \rightarrow \infty} \frac{\left\|E_{n+1}\right\|}{\left\|E_{n}\right\|}=\left|\lambda F_{J}(\bar{x}, \bar{y})\right|,
$$

where $\lambda F_{J}(\bar{x}, \bar{y})$ are the characteristic roots of the Jacobian matrix $F_{J}(\bar{x}, \bar{y})$ about $(0,0)$.

3 On the system $x_{n+1}=\frac{a y_{n-k}}{b+c \prod_{i=0}^{k} x_{n-i}}, y_{n+1}=\frac{a_{1} x_{n-k}}{b_{1}+c_{1} \prod_{i=0}^{k} y_{n-i}}$

In this section, we shall investigate the qualitative behavior of system (2). Let $(\bar{x}, \bar{y})$ be an equilibrium point of system (2), then system (2) has a unique equilibrium point $(0,0)$. To construct the corresponding linearized form of system (2), we consider the following transformation:

$$
\left(x_{n}, x_{n-1}, x_{n-2}, \ldots, x_{n-k}, y_{n}, y_{n-1}, \ldots, y_{n-k}\right) \mapsto\left(f, f_{1}, \ldots, f_{n-k}, g, g_{1}, \ldots, g_{n-k}\right),
$$

$f=\frac{a y_{n-k}}{b+c \prod_{i=0}^{k} x_{n-i}}, f_{1}=x_{n}, f_{2}=x_{n-1}, \ldots, f_{n-k}=x_{n-(k-1)}$ and $g=\frac{a_{1} x_{n-k}}{b_{1}+c_{1} \prod_{i=0}^{k} y_{n-i}}, g_{1}=y_{n}, g_{2}=y_{n-1}, \ldots$, $g_{n-k}=y_{n-(k-1)}$. The Jacobian matrix about the fixed point $(\bar{x}, \bar{y})$ under the transformation (13) is given by

$$
F_{J}(\bar{x}, \bar{y})=\left(\begin{array}{cccccccccc}
A & A & \ldots & A & A & 0 & 0 & \ldots & 0 & B \\
1 & 0 & \ldots & 0 & 0 & 0 & 0 & \ldots & 0 & 0 \\
\vdots & \vdots & \ddots & \vdots & \vdots & \vdots & \ddots & \vdots & \vdots & \vdots \\
0 & 0 & \ldots & 1 & 0 & 0 & 0 & \ldots & 0 & 0 \\
0 & 0 & \ldots & 0 & C & D & D & \ldots & D & D \\
0 & 0 & \ldots & 0 & 0 & 1 & 0 & \ldots & 0 & 0 \\
\vdots & \vdots & \ddots & \vdots & \vdots & \vdots & \ddots & \vdots & \vdots & \vdots \\
0 & 0 & \ldots & 0 & 0 & 0 & 0 & \ldots & 1 & 0
\end{array}\right),
$$

where $A=-\frac{a c \bar{y} \bar{x}^{k}}{\left(b+c \bar{x}^{k+1}\right)^{2}}, B=\frac{a}{b+c \bar{x}^{k+1}}, C=\frac{a_{1}}{b_{1}+c_{1} \bar{y}^{k+1}}$ and $D=-\frac{a_{1} c_{1} \bar{y}^{k}}{\left(b_{1}+c_{1} \bar{y}^{k+1}\right)^{2}}$.

Theorem 8 Let $\left\{\left(x_{n}, y_{n}\right)\right\}$ be a positive solution of system (2), then for every $m \geq 1$, the following results hold.

$$
\text { (i) } \quad 0 \leq x_{n} \leq \begin{cases}\left(\frac{a}{b}\right)^{m+1}\left(\frac{a_{1}}{b_{1}}\right)^{m} y_{-k} & \text { if } n=(2 k+2) m+1, \\ \left(\frac{a}{b}\right)^{m+1}\left(\frac{a_{1}}{b_{1}}\right)^{m} y_{1-k} & \text { if } n=(2 k+2) m+2, \\ \vdots & \\ \left(\frac{a}{b}\right)^{m+1}\left(\frac{a_{1}}{b_{1}}\right)^{m} y_{-1} & \text { if } n=(2 k+2) m+k, \\ \left(\frac{a}{b}\right)^{m+1}\left(\frac{a_{1}}{b_{1}}\right)^{m} y_{0} & \text { if } n=(2 k+2) m+(k+1), \\ \left(\frac{a a_{1}}{b b_{1}}\right)^{m+1} x_{-k} & \text { if } n=(2 k+2) m+(k+2), \\ \left(\frac{a a_{1}}{b b_{1}}\right)^{m+1} x_{1-k} & \text { if } n=(2 k+2) m+(k+3), \\ \vdots & \\ \left(\frac{a a_{1}}{b b_{1}}\right)^{m+1} x_{-1} & \text { if } n=(2 k+2) m+(2 k+1), \\ \left(\frac{a a_{1}}{b b_{1}}\right)^{m+1} x_{0} & \text { if } n=(2 k+2) m+(2 k+2) .\end{cases}
$$




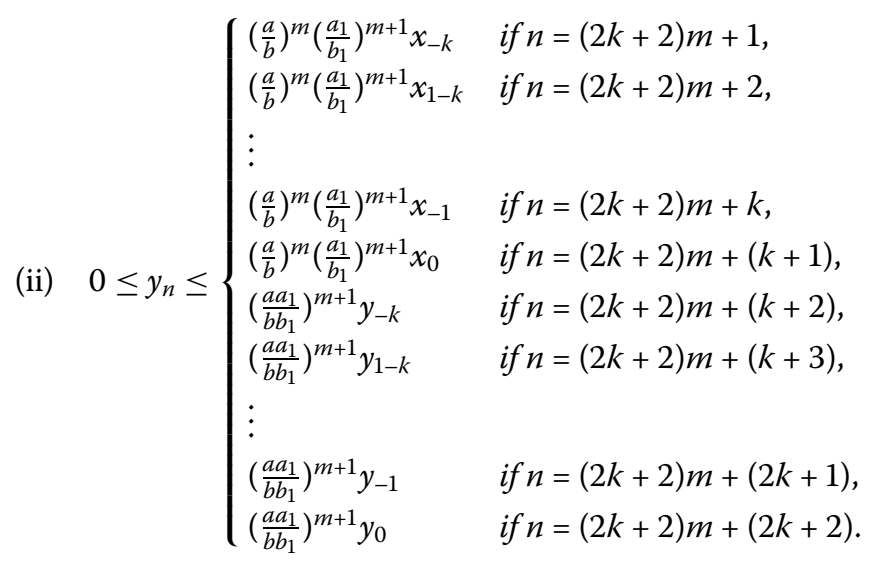

Lemma 3 Let $\frac{a a_{1}}{b b_{1}}<1$, then every solution $\left\{x_{n}, y_{n}\right\}_{n=-k}^{\infty}$ of system (2) is bounded.

Proof Assume that

$$
\lambda_{1}=\max \left\{\frac{b_{1}}{a_{1}} y_{-k}, \frac{b_{1}}{a_{1}} y_{-k+1}, \ldots, \frac{b_{1}}{a_{1}} y_{0}, x_{-k}, x_{-k+1}, \ldots, x_{0}\right\},
$$

and

$$
\lambda_{2}=\max \left\{\frac{b}{a} x_{-k}, \frac{b}{a} x_{-k+1}, \ldots, \frac{b}{a} x_{0}, y_{-k}, y_{-k+1}, \ldots, y_{0}\right\}
$$

Then from Theorem 8 one can easily see that $0 \leq x_{n}<\lambda_{1}$ and $0 \leq y_{n}<\lambda_{2}$ for all $n=$ $0,1, \ldots$

Theorem 9 The equilibrium point $(0,0)$ of equation (2) is locally asymptotically stable.

Proof The linearized system of (2) about the equilibrium point $(0,0)$ is given by

$$
X_{n+1}=F_{J}(0,0) X_{n},
$$

where

$$
X_{n}=\left(\begin{array}{c}
x_{n} \\
x_{n-1} \\
\vdots \\
x_{n-k} \\
y_{n} \\
y_{n-1} \\
\vdots \\
y_{n-k}
\end{array}\right)
$$


and

$$
H=F_{J}(0,0)=\left(\begin{array}{cccccccccc}
0 & 0 & \ldots & 0 & 0 & 0 & 0 & \ldots & 0 & \frac{a}{b} \\
1 & 0 & \ldots & 0 & 0 & 0 & 0 & \ldots & 0 & 0 \\
\vdots & \vdots & \ddots & \vdots & \vdots & \vdots & \ddots & \vdots & \vdots & \vdots \\
0 & 0 & \ldots & 1 & 0 & 0 & 0 & \ldots & 0 & 0 \\
0 & 0 & \ldots & 0 & \frac{a_{1}}{b_{1}} & 0 & 0 & \ldots & 0 & 0 \\
0 & 0 & \ldots & 0 & 0 & 1 & 0 & \ldots & 0 & 0 \\
\vdots & \vdots & \ddots & \vdots & \vdots & \vdots & \ddots & \vdots & \vdots & \vdots \\
0 & 0 & \ldots & 0 & 0 & 0 & 0 & \ldots & 1 & 0
\end{array}\right) .
$$

Let $\lambda_{1}, \lambda_{2}, \ldots, \lambda_{2 k+2}$ denote the $2 k+2$ eigenvalues of matrix $E$. Let $D=\operatorname{diag}\left(d_{1}, d_{2}, \ldots, d_{2 k+2}\right)$ be a diagonal matrix, where $d_{1}=d_{k+2}=1, d_{1+m}=d_{k+2+m}=1-m \epsilon, 1 \leq m \leq k$ and

$$
\epsilon=\min \left\{\frac{1}{k}, \frac{1}{k}\left(1-\frac{a}{b}\right), \frac{1}{k}\left(1-\frac{a_{1}}{b_{1}}\right)\right\} .
$$

Clearly, $D$ is invertible. Computing $D H D^{-1}$, we obtain

$$
D H D^{-1}=\left(\begin{array}{cccccccccc}
0 & 0 & \ldots & 0 & 0 & 0 & 0 & \ldots & 0 & \frac{a}{b} d_{1} d_{2 k+2}^{-1} \\
d_{2} d_{1}^{-1} & 0 & \ldots & 0 & 0 & 0 & 0 & \ldots & 0 & 0 \\
\vdots & \vdots & \ldots & \vdots & \vdots & \vdots & \ddots & \vdots & \vdots & \vdots \\
0 & 0 & \ldots & d_{k+1} d_{k}^{-1} & 0 & 0 & 0 & \ldots & 0 & 0 \\
0 & 0 & \ldots & 0 & \frac{a_{1}}{b_{1}} d_{k+2} d_{k+1}^{-1} & 0 & 0 & \ldots & 0 & 0 \\
0 & 0 & \ldots & 0 & 0 & d_{k+3} d_{k+2}^{-1} & 0 & \ldots & 0 & 0 \\
\vdots & \vdots & \ddots & \vdots & \vdots & \vdots & \ddots & \vdots & \vdots & \vdots \\
0 & 0 & \ldots & 0 & 0 & 0 & 0 & \ldots & d_{2 k+2} d_{2 k+1}^{-1} & 0
\end{array}\right) .
$$

Next, we have the following two inequalities:

$$
0<d_{k+1}<d_{k}<\cdots<d_{2}, \quad 0<d_{2 k+2}<d_{2 k+1}<\cdots<d_{k+3}
$$

which implies that

$$
d_{2} d_{1}^{-1}<1, \quad d_{3} d_{2}^{-1}<1, \quad \ldots, \quad d_{k+1} d_{k}^{-1}<1,
$$

and

$$
d_{k+3} d_{k+2}^{-1}<1, \quad d_{k+4} d_{k+3}^{-1}<1, \quad \ldots, \quad d_{2 k+2} d_{2 k+1}^{-1}<1 .
$$

Furthermore,

$$
\frac{a}{b} d_{1} d_{2 k+2}^{-1}=\frac{a}{b} d_{2 k+2}^{-1}=\frac{a}{b} \frac{1}{1-k \epsilon}<1,
$$

and

$$
\frac{a_{1}}{b_{1}} d_{k+2} d_{k+1}^{-1}=\frac{a_{1}}{b_{1}} d_{k+1}^{-1}=\frac{a_{1}}{b_{1}} \frac{1}{1-k \epsilon}<1 .
$$


Now $H$ has the same eigenvalues as $D H D^{-1}$, we obtain that

$$
\begin{aligned}
& \max _{1 \leq m \leq 2 k+2}\left|\lambda_{m}\right| \\
& \quad=\left\|D H D^{-1}\right\| \\
& \quad=\max \left\{d_{2} d_{1}^{-1}, \ldots, d_{k+1} d_{k}^{-1}, d_{k+3} d_{k+2}^{-1}, \ldots, d_{2 k+2} d_{2 k+1}^{-1}, \frac{a}{b} d_{1} d_{2 k+2}^{-1}, \frac{a_{1}}{b_{1}} d_{k+2} d_{k+1}^{-1}\right\}<1 .
\end{aligned}
$$

Hence, the equilibrium point $(0,0)$ of system $(2)$ is locally asymptotically stable.

Theorem 10 Let $a<b$ and $a_{1}<b_{1}$, then the equilibrium point $(0,0)$ of system (2) is globally asymptotically stable.

Proof Assume that $a<b$ and $a_{1}<b_{1}$. Then from Theorem 9 the equilibrium point $(0,0)$ of system (2) is locally asymptotically stable. Moreover, from Lemma 3 every positive solution $\left(x_{n}, y_{n}\right)$ is bounded, i.e., $0 \leq x_{n} \leq \mu$ and $0 \leq y_{n} \leq v$ for all $n=0,1,2, \ldots$, where $\mu=\max \left\{x_{-k}, x_{-k+1}, \ldots, x_{0}\right\}$ and $v=\max \left\{y_{-k}, y_{-k+1}, \ldots, y_{0}\right\}$. Now, it is sufficient to prove that $\left(x_{n}, y_{n}\right)$ is decreasing. From system (2) one has

$$
\begin{aligned}
x_{n+1} & =\frac{a y_{n-k}}{b+c \prod_{i=0}^{k} x_{n-i}} \\
& \leq \frac{a y_{n-k}}{b}<y_{n-k} .
\end{aligned}
$$

This implies that $x_{(2 k+2) n+1}<y_{(2 k+2) n-k}$ and $x_{(2 k+2) n+(2 k+3)}<y_{(2 k+2) n+(k+2)}$.

$$
\begin{aligned}
y_{n+1} & =\frac{a_{1} x_{n-k}}{b_{1}+c_{1} \prod_{i=0}^{k} y_{n-i}} \\
& \leq \frac{a x_{n-4}}{b}<x_{n-k} .
\end{aligned}
$$

This implies that

$$
y_{(2 k+2) n+1}<x_{(2 k+2) n-k} \quad \text { and } \quad y_{(2 k+2) n+(2 k+3)}<x_{(2 k+2) n+(k+2)} .
$$

Hence, $x_{(2 k+2) n+(2 k+3)}<y_{(2 k+2) n+(k+2)}<x_{(2 k+2) n+1}$ and $y_{(2 k+2) n+(2 k+3)}<x_{(2 k+2) n+(k+2)}<y_{(2 k+2) n+1}$. Hence, the subsequences

$$
\left\{x_{(2 k+2) n+1}\right\}, \quad\left\{x_{(2 k+2) n+2}\right\}, \quad \ldots, \quad\left\{x_{(2 k+2) n+(2 k+2)}\right\}
$$

and

$$
\left\{y_{(2 k+2) n+1}\right\}, \quad\left\{y_{(2 k+2) n+2}\right\}, \quad \ldots, \quad\left\{y_{(2 k+2) n+(2 k+2)}\right\}
$$

are decreasing. Therefore the sequences $\left\{x_{n}\right\}$ and $\left\{y_{n}\right\}$ are decreasing. Hence, $\lim _{n \rightarrow \infty} x_{n}=$ $\lim _{n \rightarrow \infty} y_{n}=0$. 


\subsection{Rate of convergence}

Assume that $\lim _{n \rightarrow \infty} x_{n}=\bar{x}$ and $\lim _{n \rightarrow \infty} y_{n}=\bar{y}$. First we will find a system of limiting equations for system (2). The error terms are given as

$$
\begin{aligned}
& x_{n+1}-\bar{x}=\sum_{i=0}^{k} A_{i}\left(x_{n-i}-\bar{x}\right)+\sum_{i=0}^{k} B_{i}\left(y_{n-i}-\bar{y}\right), \\
& y_{n+1}-\bar{y}=\sum_{i=0}^{k} C_{i}\left(x_{n-i}-\bar{x}\right)+\sum_{i=0}^{k} D_{i}\left(y_{n-i}-\bar{y}\right) .
\end{aligned}
$$

Set $e_{n}^{1}=x_{n}-\bar{x}$ and $e_{n}^{2}=y_{n}-\bar{y}$, then one has

$$
\begin{aligned}
& e_{n+1}^{1}=\sum_{i=0}^{k} A_{i} e_{n-i}^{1}+\sum_{i=0}^{k} B_{i} e_{n-i}^{2}, \\
& e_{n+1}^{2}=\sum_{i=0}^{k} C_{i} e_{n-i}^{1}+\sum_{i=0}^{k} D_{i} e_{n-i}^{2},
\end{aligned}
$$

where

$$
\begin{gathered}
A_{0}=-\frac{a c \bar{y} x_{n-1} x_{n-2} \cdots x_{n-k}}{\left(b+c \prod_{i=0}^{k} x_{n-i}\right)\left(b+c \bar{x}^{k+1}\right)}, \quad A_{1}=-\frac{a c \bar{x} \bar{y} x_{n-2} x_{n-3} \cdots x_{n-k}}{\left(b+c \prod_{i=0}^{k} x_{n-i}\right)\left(b+c \bar{x}^{k+1}\right)}, \\
A_{k-1}=-\frac{a c \bar{x}^{k-1} \bar{y} x_{n-k}}{\left(b+c \prod_{i=0}^{k} x_{n-i}\right)\left(b+c \bar{x}^{k+1}\right)}, \quad A_{k}=-\frac{a c \bar{x}^{k} \bar{y}}{\left(b+c \prod_{i=0}^{k} x_{n-i}\right)\left(b+c \bar{x}^{k+1}\right)}, \\
B_{i}=0 \text { for } i \in\{0,1, \ldots, k-1\}, B_{k}=\frac{a}{b+c \prod_{i=0}^{k} x_{n-i}}, C_{i}=0 \text { for } i \in\{0,1, \ldots, k-1\}, \\
C_{k}=\frac{a_{1}}{b_{1}+c_{1} \prod_{i=0}^{k} y_{n-i}}, \quad D_{0}=-\frac{a_{1} c_{1} \bar{x} y_{n-1} y_{n-2} \cdots y_{n-k}}{\left(b_{1}+c_{1} \prod_{i=0}^{k} y_{n-i}\right)\left(b_{1}+c_{1} \bar{y}^{k+1}\right)}, \\
D_{1}=-\frac{a_{1} c_{1} \bar{x} \bar{y} y_{n-2} y_{n-3} \cdots y_{n-k}}{\left(b_{1}+c_{1} \prod_{i=0}^{k} y_{n-i}\right)\left(b_{1}+c_{1} \bar{y}^{k+1}\right)}, \\
D_{k-1}=-\frac{a_{1} c_{1} \bar{x} \bar{y}^{k-1} y_{n-k}}{\left(b_{1}+c_{1} \prod_{i=0}^{k} y_{n-i}\right)\left(b_{1}+c_{1} \bar{y}^{k+1}\right)},
\end{gathered}
$$

and $D_{k}=-\frac{a_{1} c_{1} \bar{y} \bar{y}^{k}}{\left(b_{1}+c_{1} \prod_{i=0}^{k} y_{n-i}\right)\left(b_{1}+c_{1} \bar{y}^{k+1}\right)}$. Taking the limits, we obtain $\lim _{n \rightarrow \infty} A_{i}=-\frac{a c \bar{y} \bar{x}^{k}}{\left(b+c \bar{x}^{k+1}\right)^{2}}$ for $i \in\{0,1, \ldots, k\}, \lim _{n \rightarrow \infty} B_{i}=0$ for $i \in\{0,1, \ldots, k-1\}, \lim _{n \rightarrow \infty} B_{k}=\frac{a}{b+c \bar{x}^{k+1}}, \lim _{n \rightarrow \infty} C_{i}=0$ for $i \in\{0,1, \ldots, k-1\}, \lim _{n \rightarrow \infty} C_{k}=\frac{a_{1}}{b_{1}+c_{1} \bar{y}^{k+1}}, \lim _{n \rightarrow \infty} D_{i}=-\frac{a_{1} c_{1} \bar{x} \bar{y}^{k}}{\left(b_{1}+c_{1} \bar{y}^{k+1}\right)^{2}}$ for $i \in\{0,1, \ldots, k\}$, So, the limiting system of error terms can be written as

$$
E_{n+1}=K E_{n}
$$


where

$$
E_{n}=\left(\begin{array}{c}
e_{n}^{1} \\
e_{n-1}^{1} \\
\vdots \\
e_{n-k}^{1} \\
e_{n}^{2} \\
e_{n-1}^{2} \\
\vdots \\
e_{n-k}^{2}
\end{array}\right)
$$

and

$$
K=\left(\begin{array}{cccccccccc}
0 & 0 & \ldots & 0 & 0 & 0 & 0 & \ldots & 0 & \frac{a}{b} \\
1 & 0 & \ldots & 0 & 0 & 0 & 0 & \ldots & 0 & 0 \\
\vdots & \vdots & \ddots & \vdots & \vdots & \vdots & \ddots & \vdots & \vdots & \vdots \\
0 & 0 & \ldots & 1 & 0 & 0 & 0 & \ldots & 0 & 0 \\
0 & 0 & \ldots & 0 & \frac{a_{1}}{b_{1}} & 0 & 0 & \ldots & 0 & 0 \\
0 & 0 & \ldots & 0 & 0 & 1 & 0 & \ldots & 0 & 0 \\
\vdots & \vdots & \ddots & \vdots & \vdots & \vdots & \ddots & \vdots & \vdots & \vdots \\
0 & 0 & \ldots & 0 & 0 & 0 & 0 & \ldots & 1 & 0
\end{array}\right),
$$

which is similar to the linearized system of (2) about the equilibrium point $(\bar{x}, \bar{y})=(0,0)$. Using proposition (1), one has the following result.

Theorem 11 Assume that $\left\{\left(x_{n}, y_{n}\right)\right\}$ is a positive solution of system (2) such that $\lim _{n \rightarrow \infty} x_{n}=$ $\bar{x}$, and $\lim _{n \rightarrow \infty} y_{n}=\bar{y}$, where $(\bar{x}, \bar{y})=(0,0)$. Then the error vector $E_{n}$ of every solution of $(2)$ satisfies both of the following asymptotic relations:

$$
\lim _{n \rightarrow \infty}\left(\left\|E_{n}\right\|\right)^{\frac{1}{n}}=\left|\lambda F_{J}(\bar{x}, \bar{y})\right|, \quad \lim _{n \rightarrow \infty} \frac{\left\|E_{n+1}\right\|}{\left\|E_{n}\right\|}=\left|\lambda F_{J}(\bar{x}, \bar{y})\right|,
$$

where $\lambda F_{J}(\bar{x}, \bar{y})$ are the characteristic roots of the Jacobian matrix $F_{J}(\bar{x}, \bar{y})$ about $(\bar{x}, \bar{y})=$ $(0,0)$.

\section{Examples}

In order to verify our theoretical results, we consider some interesting numerical examples in this section. These examples show that the equilibrium point $(0,0)$ of both systems $(1)$ and (2) is globally asymptotically stable.

Example 1 Consider system (1) with initial conditions $x_{-8}=1.9, x_{-7}=1.7, x_{-6}=2.5, x_{-5}=$ $0.9, x_{-4}=1.5, x_{-3}=10.4, x_{-2}=6.9, x_{-1}=0.6, x_{0}=2.9, y_{-8}=2.8, y_{-7}=1.6, y_{-6}=1.8, y_{-5}=$ 2.6, $y_{-4}=2.8, y_{-3}=2.8, y_{-2}=3.5, y_{-1}=2.1, y_{0}=1.6$. Moreover, choose the parameters $\alpha=15.5, \beta=17, \gamma=27, \alpha_{1}=11.2, \beta_{1}=12, \gamma_{1}=23$. Then system (1) can be written as

$$
x_{n+1}=\frac{15.5 x_{n-8}}{17+27 \prod_{i=0}^{8} y_{n-i}}, \quad y_{n+1}=\frac{11.2 y_{n-8}}{12+23 \prod_{i=0}^{8} x_{n-i}}, \quad n=0,1, \ldots
$$


with initial conditions $x_{-8}=1.9, x_{-7}=1.7, x_{-6}=2.5, x_{-5}=0.9, x_{-4}=1.5, x_{-3}=10.4, x_{-2}=$ 6.9, $x_{-1}=0.6, x_{0}=2.9, y_{-8}=2.8, y_{-7}=1.6, y_{-6}=1.8, y_{-5}=2.6, y_{-4}=2.8, y_{-3}=2.8, y_{-2}=$ $3.85, y_{-1}=2.1, y_{0}=1.6$. Moreover, in Figure 1, the plot of $x_{n}$ is shown in Figure 1a, the plot of $y_{n}$ is shown in Figure 1b, and an attractor of system (14) is shown in Figure 1c.

Example 2 Consider system (1) with initial conditions $x_{-15}=0.9, x_{-14}=1.9, x_{-13}=0.8$, $x_{-12}=0.3, x_{-11}=1.7, x_{-10}=2.9, x_{-9}=1.9, x_{-8}=2.9, x_{-7}=0.3, x_{-6}=3.5, x_{-5}=1.4, x_{-4}=$ $1.5, x_{-3}=13.4, x_{-2}=17.9, x_{-1}=11.7, x_{0}=9.8, y_{-15}=2.9, y_{-14}=3.8, y_{-13}=0.9, y_{-12}=0.8$, $y_{-11}=1.1, y_{-10}=3.9, y_{-9}=3.6, y_{-8}=1.9, y_{-7}=3.7, y_{-6}=2.9, y_{-5}=1.5, y_{-4}=0.9, y_{-3}=1.8$, $y_{-2}=3.9, y_{-1}=6.7, y_{0}=2.9$. Moreover, choose the parameters $\alpha=120, \beta=125, \gamma=11.5$, $\alpha_{1}=140, \beta_{1}=145, \gamma_{1}=14.5$. Then system (1) can be written as

$$
x_{n+1}=\frac{120 x_{n-15}}{125+11.5 \prod_{i=0}^{15} y_{n-i}}, \quad y_{n+1}=\frac{140 y_{n-15}}{145+14.5 \prod_{i=0}^{15} x_{n-i}}, \quad n=0,1, \ldots,
$$

with initial conditions $x_{-15}=0.9, x_{-14}=1.9, x_{-13}=0.8, x_{-12}=0.3, x_{-11}=1.7, x_{-10}=2.9$, $x_{-9}=1.9, x_{-8}=2.9, x_{-7}=0.3, x_{-6}=3.5, x_{-5}=1.4, x_{-4}=1.5, x_{-3}=13.4, x_{-2}=17.9, x_{-1}=11.7$, $x_{0}=9.8, y_{-15}=2.9, y_{-14}=3.8, y_{-13}=0.9, y_{-12}=0.8, y_{-11}=1.1, y_{-10}=3.9, y_{-9}=3.6, y_{-8}=1.9$, $y_{-7}=3.7, y_{-6}=2.9, y_{-5}=1.5, y_{-4}=0.9, y_{-3}=1.8, y_{-2}=3.9, y_{-1}=6.7, y_{0}=2.9$. Moreover, in Figure 2, the plot of $x_{n}$ is shown in Figure 2a, the plot of $y_{n}$ is shown in Figure 2b, and an attractor of system (15) is shown in Figure 2c.

Example 3 Consider system (2) with initial conditions $x_{-6}=0.9, x_{-5}=2.9, x_{-4}=1.6, x_{-3}=$ 9.4, $x_{-2}=6.9, x_{-1}=2.8, x_{0}=1.9, y_{-6}=1.9, y_{-5}=2.7, y_{-4}=2.9, y_{-3}=2.8, y_{-2}=3.9, y_{-1}=$ 2.4, $y_{0}=0.8$. Moreover, choose the parameters $a=115, b=117, c=27, a_{1}=111, b_{1}=112$, $c_{1}=23$. Then system (2) can be written as

$$
x_{n+1}=\frac{115 y_{n-6}}{117+27 \prod_{i=0}^{6} x_{n-i}}, \quad y_{n+1}=\frac{111 x_{n-6}}{112+23 \prod_{i=0}^{6} y_{n-i}}, \quad n=0,1, \ldots
$$

with initial conditions $x_{-6}=0.9, x_{-5}=2.9, x_{-4}=1.6, x_{-3}=9.4, x_{-2}=6.9, x_{-1}=2.8, x_{0}=1.9$, $y_{-6}=1.9, y_{-5}=2.7, y_{-4}=2.9, y_{-3}=2.8, y_{-2}=3.9, y_{-1}=2.4, y_{0}=0.8$. Moreover, in Figure 3, the plot of $x_{n}$ is shown in Figure 3a, the plot of $y_{n}$ is shown in Figure 3b, and an attractor of system (16) is shown in Figure 3c.

Example 4 Consider system (2) with initial conditions $x_{-12}=7.1, x_{-11}=2.8, x_{-10}=19.1$, $x_{-9}=20.9, x_{-8}=9.9, x_{-7}=13.4, x_{-6}=1.9, x_{-5}=10.9, x_{-4}=12.9, x_{-3}=0.9, x_{-2}=1.9, x_{-1}=$ $15.8, x_{0}=4.9, y_{-12}=10.9, y_{-11}=1.1, y_{-10}=3.7, y_{-9}=0.6, y_{-8}=1.7, y_{-7}=1.7, y_{-6}=1.9, y_{-5}=$ $1.9, y_{-4}=1.9, y_{-3}=2.8, y_{-2}=17.9, y_{-1}=27.4, y_{0}=0.04$. Moreover, choose the parameters $a=121, b=125, c=9, a_{1}=129, b_{1}=130, c_{1}=7$. Then system (2) can be written as

$$
x_{n+1}=\frac{121 y_{n-12}}{125+9 \prod_{i=0}^{12} x_{n-i}}, \quad y_{n+1}=\frac{129 x_{n-12}}{130+7 \prod_{i=0}^{12} y_{n-i}}, \quad n=0,1, \ldots
$$

with initial conditions $x_{-12}=7.1, x_{-11}=2.8, x_{-10}=19.1, x_{-9}=20.9, x_{-8}=9.9, x_{-7}=13.4$, $x_{-6}=1.9, x_{-5}=10.9, x_{-4}=12.9, x_{-3}=0.9, x_{-2}=1.9, x_{-1}=15.8, x_{0}=4.9, y_{-12}=10.9, y_{-11}=$ $1.1, y_{-10}=3.7, y_{-9}=0.6, y_{-8}=1.7, y_{-7}=1.7, y_{-6}=1.9, y_{-5}=1.9, y_{-4}=1.9, y_{-3}=2.8, y_{-2}=$ 17.9, $y_{-1}=27.4, y_{0}=0.04$. Moreover, in Figure 4, the plot of $x_{n}$ is shown in Figure 4a, the plot of $y_{n}$ is shown in Figure $4 \mathrm{~b}$, and an attractor of system (17) is shown in Figure 4c. 


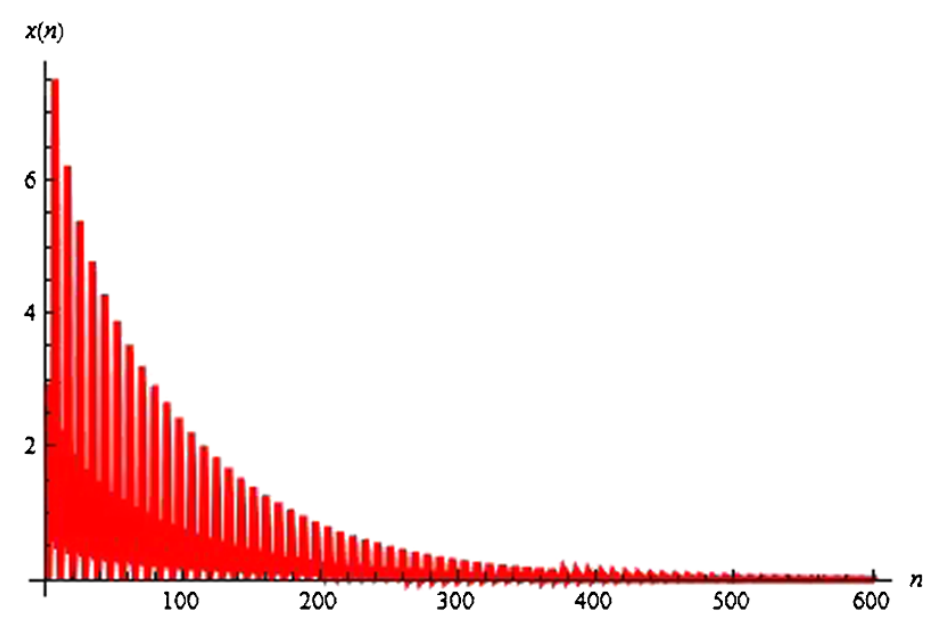

(a) Plot of $x_{n}$ for system (14).

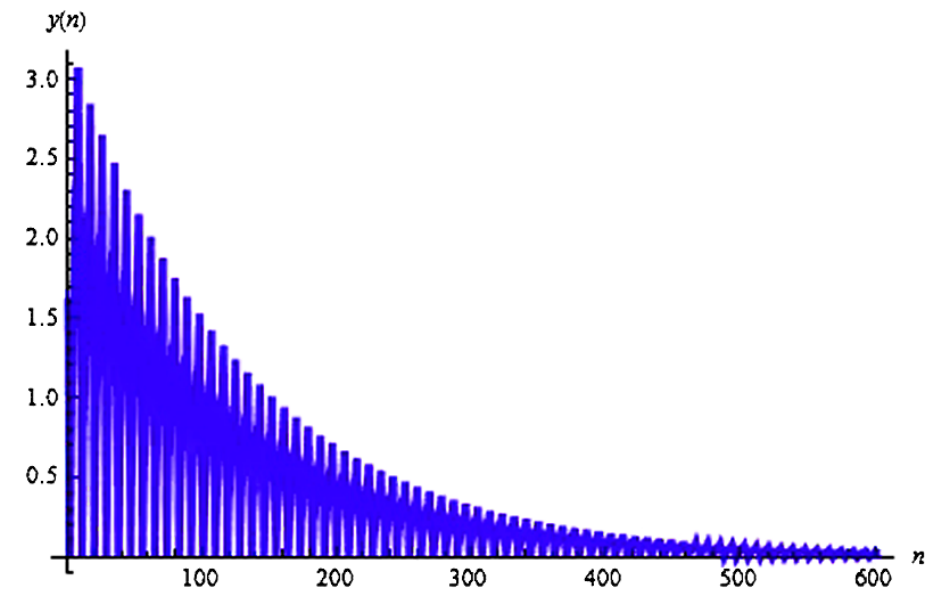

(b) Plot of $y_{n}$ for system (14).

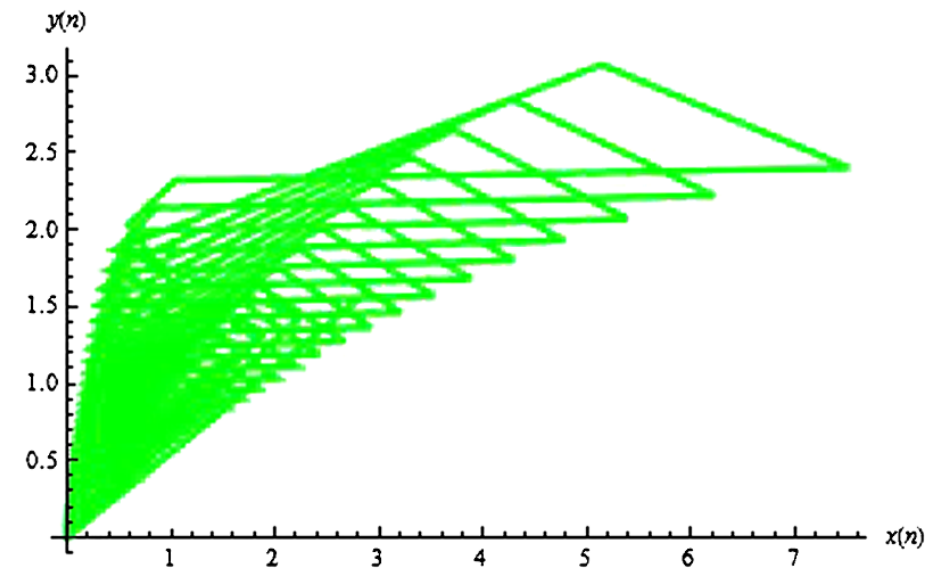

(c) An attractor of system (14).

Figure 1 Plots for system (14). 


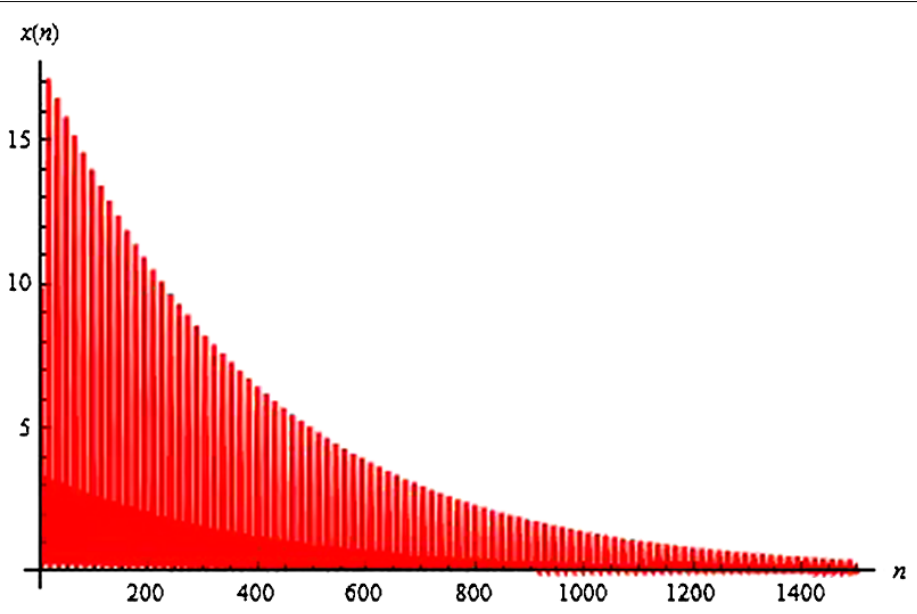

(a) Plot of $x_{n}$ for system (15).

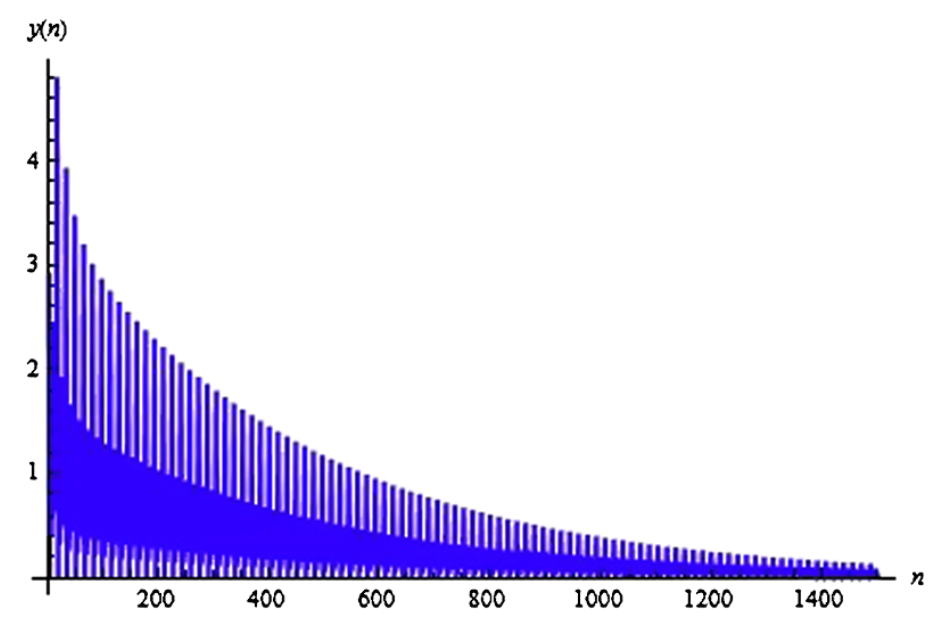

(b) Plot of $y_{n}$ for system (15).

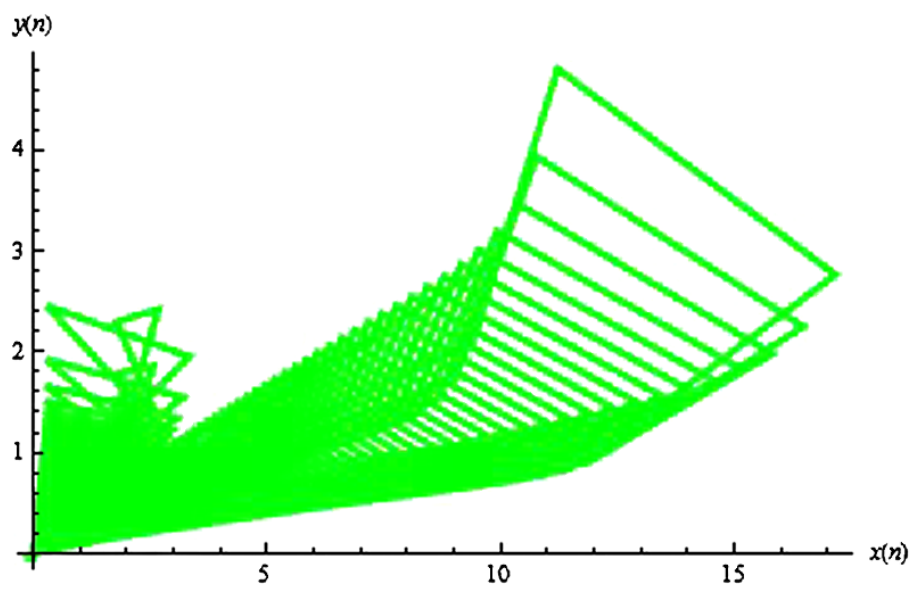

(c) An attractor of system (15).

Figure 2 Plots for system (15). 


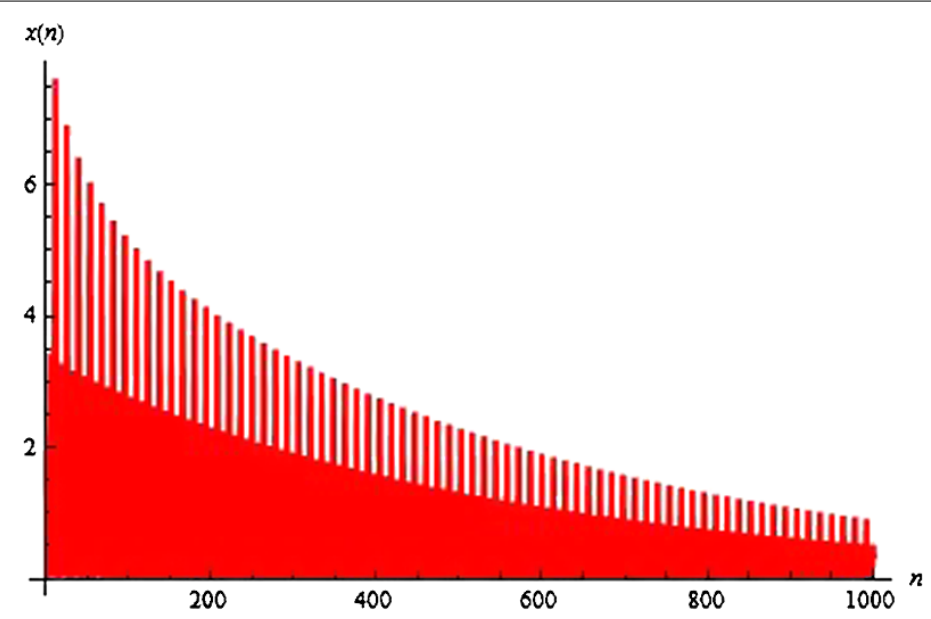

(a) Plot of $x_{n}$ for system (16).

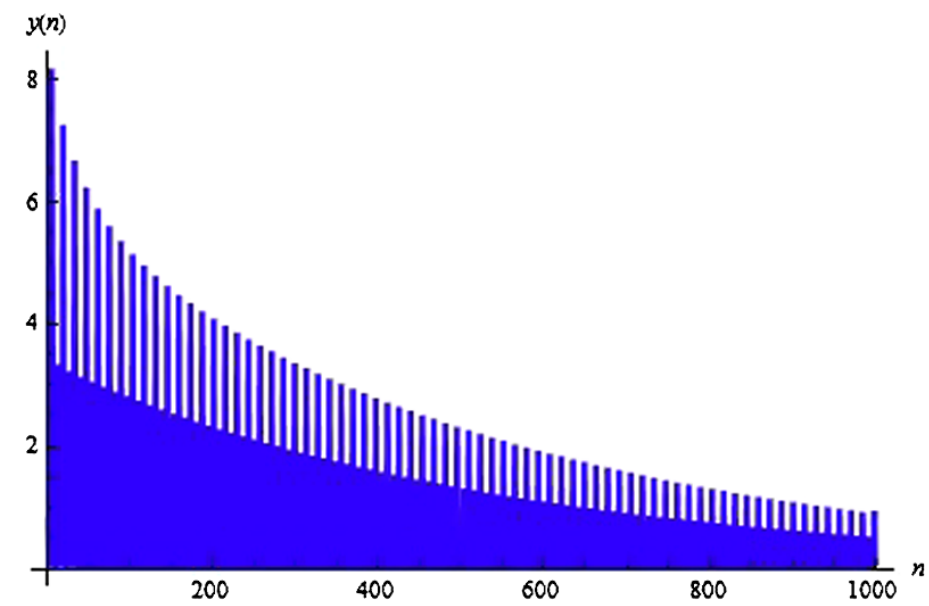

(b) Plot of $y_{n}$ for system (16).

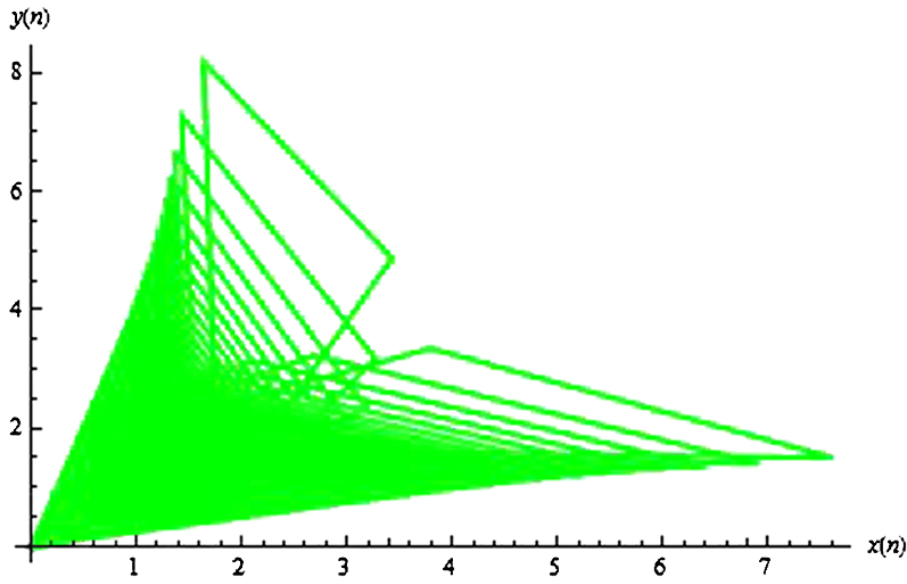

(c) An attractor of system (16).

Figure 3 Plots for system (16). 


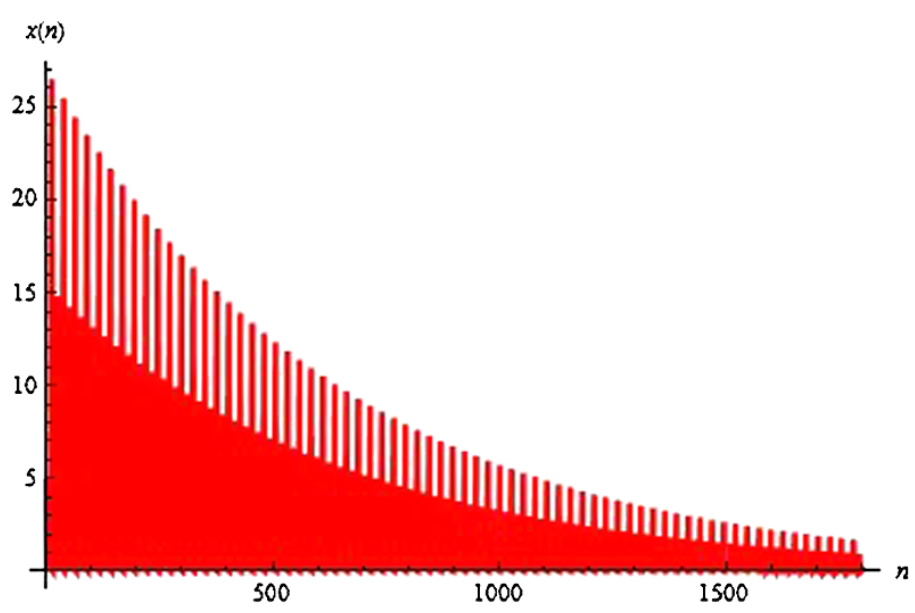

(a) Plot of $x_{n}$ for system (17).

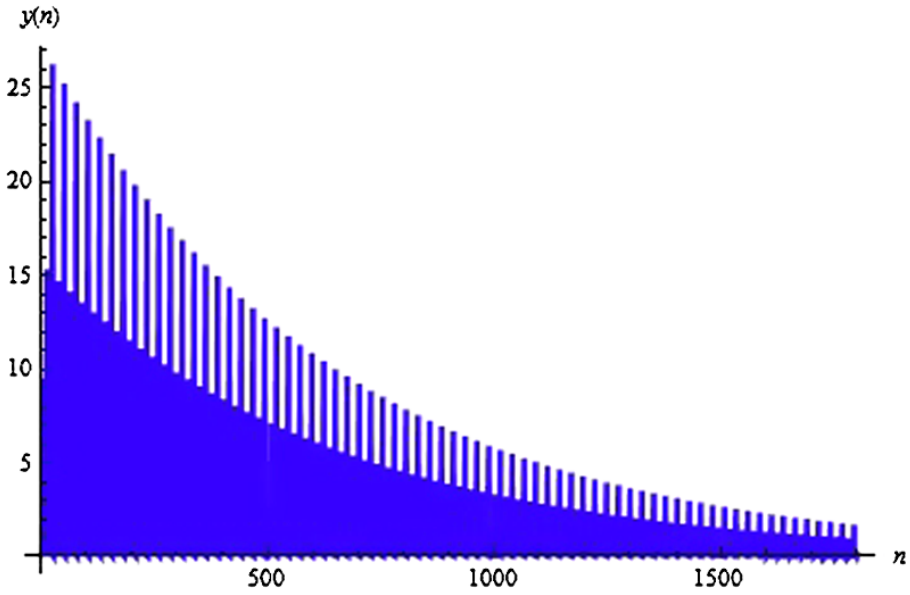

(b) Plot of $y_{n}$ for system (17).

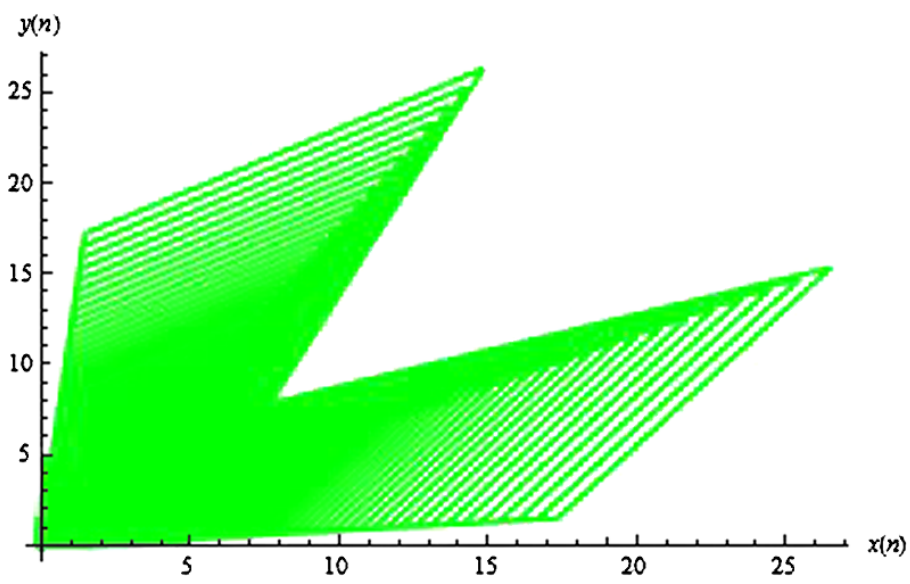

(c) An attractor of system (17).

Figure 4 Plots for system (17). 


\section{Conclusion}

This work is a natural extension of $[11,20,21]$. In the paper, we have investigated the qualitative behavior of $(2 k+2)$-dimensional discrete dynamical systems. Each system has only one equilibrium point which is stable under some restriction to parameters. The linearization method is used to show that equilibrium point $(0,0)$ is locally asymptotically stable. The main objective of dynamical systems theory is to predict the global behavior of a system based on the knowledge of its present state. An approach to this problem consists of determining the possible global behaviors of the system and determining which initial conditions lead to these long-term behaviors. In case of higher-order dynamical systems, it is crucial to discuss global behavior of the system. Some powerful tools such as semiconjugacy and weak contraction cannot be used to analyze global behavior of systems (1) and (2). In the paper, we prove the global asymptotic stability of equilibrium point $(0,0)$ by using simple techniques. We have carried out a systematical local and global stability analysis of both systems. The most important finding here is that the unique equilibrium point $(0,0)$ can be a global asymptotic attractor for systems (1) and (2). Moreover, we have determined the rate of convergence of a solution that converges to the equilibrium point $(0,0)$ of systems (1) and (2). Some numerical examples are provided to support our theoretical results. These examples are experimental verifications of theoretical discussions.

\section{Competing interests}

The authors have no competing interests.

Authors' contributions

All authors contributed equally in drafting this manuscript and giving the main proofs.

\section{Acknowledgements}

This work was supported by the Higher Education Commission of Pakistan.

Received: 3 October 2013 Accepted: 7 November 2013 Published: 03 Dec 2013

References

1. Cinar, C: On the positive solutions of the difference equation system $x_{n+1}=\frac{1}{y_{n}} ; y_{n+1}=\frac{y_{n}}{x_{n-1} y_{n-1}}$. Appl. Math. Comput. 158, 303-305 (2004)

2. Stević, S: On some solvable systems of difference equations. Appl. Math. Comput. 218, 5010-5018 (2012)

3. Kurbanli, AS: On the behavior of positive solutions of the system of rational difference equations $x_{n+1}=\frac{x_{n-1}}{y_{n} x_{n-1}-1}$, $y_{n+1}=\frac{y_{n-1}}{x_{n} y_{n-1}-1}, z_{n+1}=\frac{1}{y_{n} z_{n}}$. J. Differ. Equ. 2011, 40 (2011)

4. Stević, S: On a third-order system of difference equations. Appl. Math. Comput. 218, 7649-7654 (2012)

5. Bajo, I, Liz, E: Global behaviour of a second-order nonlinear difference equation. J. Differ. Equ. Appl. 17(10), 1471-1486 (2011)

6. Kalabusiśc, S, Kulenović, MRS, Pilav, E: Dynamics of a two-dimensional system of rational difference equations of Leslie-Gower type. Adv. Differ. Equ. (2011). doi:10.1186/1687-1847-2011-29

7. Kalabusicić, S, Kulenović, MRS, Pilav, E: Global dynamics of a competitive system of rational difference equations in the plane. Adv. Differ. Equ. 2009, Article ID 132802 (2009)

8. Kurbanli, AS, Çinar, C, Yalçinkaya, I: On the behavior of positive solutions of the system of rational difference equations $x_{n+1}=\frac{x_{n-1}}{y_{n} x_{n-1}+1} \cdot y_{n+1}=\frac{y_{n-1}}{x_{n} y_{n-1}+1}$. Math. Comput. Model. 53, 1261-1267 (2011)

9. Din, Q: Dynamics of a discrete Lotka-Volterra model. Adv. Differ. Equ. 1, 1-13 (2013)

10. Din, Q, Donchev, T: Global character of a host-parasite model. Chaos Solitons Fractals 54, 1-7 (2013)

11. Din, Q, Qureshi, MN, Khan, AQ: Dynamics of a fourth-order system of rational difference equations. Adv. Differ. Equ. 1, 1-15 (2012)

12. Din, Q, Khan, AQ, Qureshi, MN: Qualitative behavior of a host-pathogen model. Adv. Differ. Equ. (2013). doi:10.1186/1687-1847-2013-263

13. Din, Q: Global behavior of a rational difference equation. Acta Univ. Apulensis 34, 35-49 (2013)

14. Din, Q, Ibrahim, TF: Global behavior of a neural networks system. Indian J. Comput. Appl. Math. 1(1), $79-92$ (2013)

15. Qureshi, MN, Khan, AQ, Din, Q: Global behavior of third order system of rational difference equations. Int. J. Eng. Res. Technol. 2(5), 2182-2191 (2013)

16. El-Metwally, H, Elsayed, EM: Form of solutions and periodicity for systems of difference equations. J. Comput. Anal. Appl. 15(5), 852-857 (2013)

17. Touafek, N, Elsayed, EM: On the solutions of systems of rational difference equations. Math. Comput. Model. 55, 1987-1997 (2012) 
18. Elsayed, EM, El-Metwally, HA: On the solutions of some nonlinear systems of difference equations. Adv. Differ. Equ. (2013). doi:10.1186/1687-1847-2013-161

19. Elsayed, EM: Solution and attractivity for a rational recursive sequence. Discrete Dyn. Nat. Soc. 2011, Article ID 982309 (2011)

20. Zhang, Q, Yang, L, Liu, J: Dynamics of a system of rational third order difference equation. J. Differ. Equ. (2012). doi:10.1186/1687-1847-2012-136

21. Shojaei, M, Saadati, R, Adibi, H: Stability and periodic character of a rational third order difference equation. Chaos Solitons Fractals 39, 1203-1209 (2009)

22. Sedaghat, H: Nonlinear Difference Equations: Theory with Applications to Social Science Models. Kluwer Academic, Dordrecht (2003)

23. Kocic, VL, Ladas, G: Global Behavior of Nonlinear Difference Equations of Higher Order with Applications. Kluwer Academic, Dordrecht (1993)

24. Pituk, M: More on Poincare's and Perron's theorems for difference equations. J. Differ. Equ. Appl. 8, 201-216 (2002)

10.1186/1687-1847-2013-354

Cite this article as: Khan et al:: Global dynamics of some systems of higher-order rational difference equations.

Advances in Difference Equations 2013, 2013:354

Submit your manuscript to a SpringerOpen ${ }^{\circ}$ journal and benefit from:

- Convenient online submission

- Rigorous peer review

- Immediate publication on acceptance

- Open access: articles freely available online

- High visibility within the field

- Retaining the copyright to your article

Submit your next manuscript at $>$ springeropen.com 